\title{
Ki-1 (CD30)-positive Anaplastic Large Cell Lymphoma, Sarcomatoid Variant Accompanied by Spontaneously Regressing Lymphadenopathy
}

\author{
Kosuke Kashimabara, Hiroyuki Nakamura, Hiroshi Kusama*, Koji Kishi, \\ Hisanaga Yagyu, Satoshi TAKagi and Takeshi Matsuoka
}

\begin{abstract}
Although it has been reported that primary Ki-1 (CD30)-positive anaplastic large cell lymphoma (ALCL) of the skin may undergo spontaneous regression, it is rare for ALCL without cutaneous involvement to have spontaneously regressing lymphadenopathy. We report a case of sarcomatoid variant of ALCL accompanied by spontaneously regressing lymphadenopathy. The patient had gastric and pulmonary involvement of ALCL in addition to systemic lymphadenopathy, but with no cutaneous involvement. The lymphadenopathy spontaneously improved gradually during a period of one month without any treatment. At the same time, multiple small nodules in both lung fields decreased on chest computed tomography and multiple elevated gastric tumors with dimples were endoscopically recognized to have improved. He has since been treated with combination chemotherapy because of recurrence of the lymphadenopathy.
\end{abstract}

(Internal Medicine 38: 49-53, 1999)

Key words: $\mathrm{Ki}-1$ (CD30) antigen, storiform growth pattern, pulmonary and gastric involvement, soluble interleukin-2 receptor level

\section{Introduction}

Anaplastic large cell lymphoma (ALCL) was identified as a distinct morphological entity by Stein et al (1) in 1985. This is a high-grade malignant lymphoma with morphologic features of large cells, including a Reed-Sternberg-like cell appearance, and the expression of the Ki-1 (CD30) antigen or epithelial membrane antigen (EMA) (1,2). Although it has been reported that primary ALCL of the skin may undergo spontaneous regression (3-5), there is only one report in which ALCL without cutaneous involvement showed spontaneous regression of lymphadenopathy (6). The present report describes a 44year-old man with a Ki-1 (CD30)-positive ALCL accompanied by spontaneously regressing lymphadenopathy.

\section{Case Report}

A 44-year-old smoking man was admitted for evaluation of multiple small nodular shadows in both lung fields in September 1997. He had been in good health until three weeks previ- ously when he had a fever of unknown cause and a mass $(40 \times 30$ $\mathrm{mm}$ ) in the left inguinal region. On admission, his temperature was $37.8^{\circ} \mathrm{C}$, heart rate 100 beat $/ \mathrm{min}$, respiratory rate $16 / \mathrm{min}$, and blood pressure $138 / 90 \mathrm{mmHg}$. Fine crackles were audible over both lung fields. Arterial blood gas analysis revealed slight hypoxemia (pH 7.48, partial pressure of oxygen $\left(\mathrm{PaO}_{2}\right) 65.3$ Torr, partial pressure of carbon dioxide $\left(\mathrm{PaCO}_{2}\right) 36.9$ Torr, $\mathrm{HCO}_{3} 26.7 \mathrm{mmol} / \mathrm{l}$ ). The results of blood counts and blood chemistry tests were normal, except for increased levels of white blood cells $\left(15,000 / \mathrm{mm}^{3}\right)$, alkaline phosphatase (393 U/ l) and C-reactive protein $(6.19 \mathrm{mg} / \mathrm{dl})$. Antinuclear, anti-DNA antibody, rheumatoid factor, and anti-varicella zoster virus, cytomegalovirus immunoglobulin M (IgM) and Epstein-Barr virus (EBV) antibody were negative. The soluble interleukin2 receptor (IL-2R) level was $5,720 \mathrm{U} / \mathrm{ml}$.

A computed tomography (CT) of the chest revealed hilar and mediastinal lymphadenopathy, and multiple small nodular shadows in both lung fields (Fig. 1A). A CT of the abdomen revealed paraaortic lymphadenopathy (Fig. 1B). A ${ }^{67} \mathrm{Ga}$ scintigram showed an abnormal accumulation in the stomach, and

From the Fifth Department of Internal Medicine, Tokyo Medical College, Ibaraki and *the Department of Pathology, Tokyo Medical College Kasumigaura Hospital, Ibaraki

Received for publication June 1, 1998; Accepted for publication October 16, 1998

Reprint requests should be addressed to Dr. Kosuke Kashiwabara, the Fifth Department of Internal Medicine, Tokyo Medical College, 3-20-1 Ami, Inashiki-gun, Ibaraki 300-0395 
in the left supraclavicular and inguinal regions.

Histologically, sections from the inguinal mass showed a proliferation of large oval tumor cells which were pleomorphic and had abundant cytoplasm, and the presence of an abundant extracellular myxoid matrix (Fig. 2A). Some of the neoplastic cells were spindle-shaped with a storiform growth pattern (Fig. 2B). Immunohistochemical studies showed staining of tumor cells by Ki-1/Ber-H2 (CD30), UCHL-1 (CD45RO) and EMA and negative stain with leukocyte common antigen (LCA: CD45RB), cytokeratin and L26 (CD20). The stain of Ki-1/Ber$\mathrm{H} 2$ was localized to the cell membrane, Golgi zone, or both in tumor cells (Fig. 2C). EBV-encoded small RNA (EBER-1) in situ hybridization was not detected. Analysis of T cell receptor $\beta$-chain and immunoglobulin heavy chain $\mathrm{J}_{\mathrm{H}}$ rearrangements

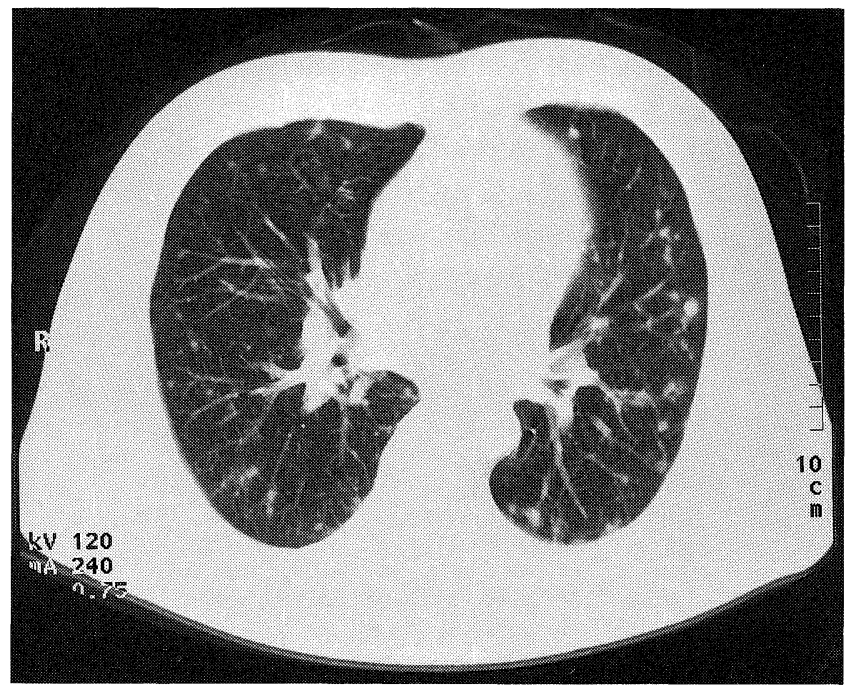

A

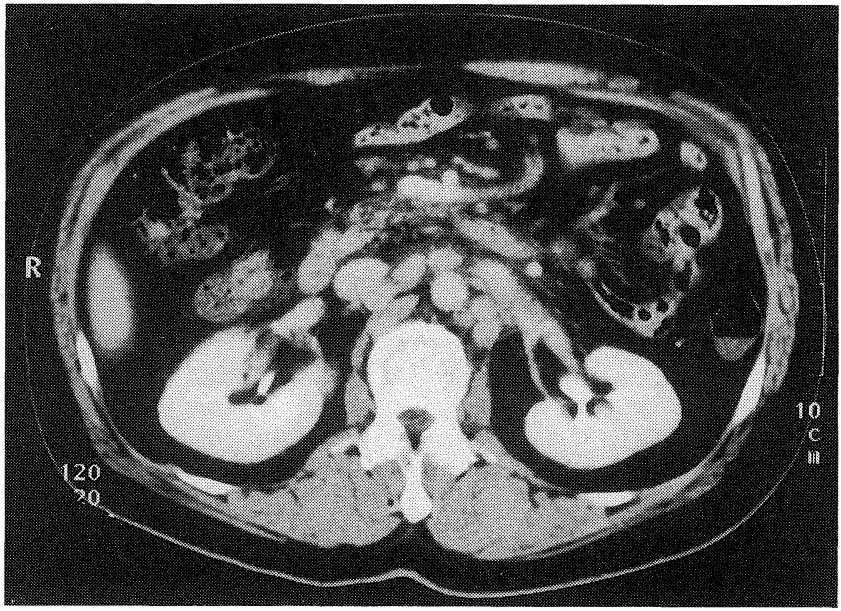

$\mathrm{B}$

Figure 1. A computed tomography (CT) of the chest reveals multiple small nodular shadows and ground-glass opacities in both lower lung fields (A). A CT of the abdomen reveals paraaortic lymphadenopathy (B).

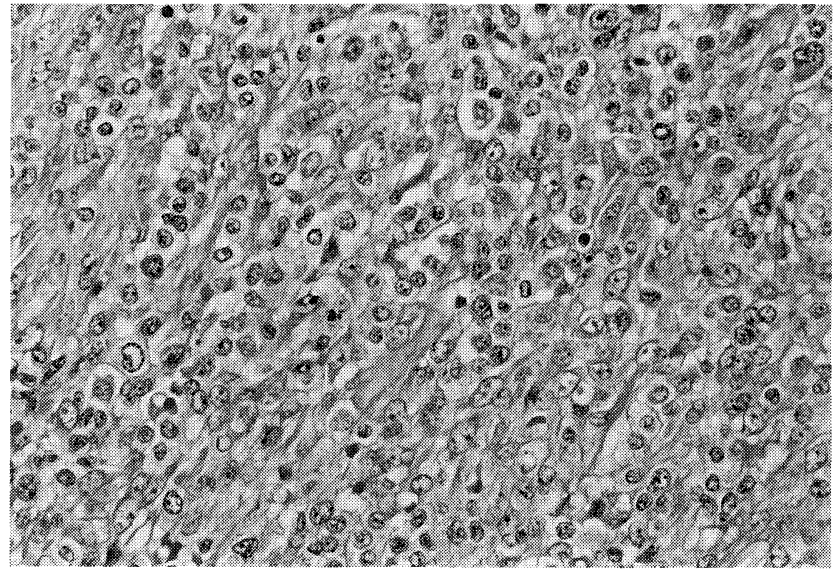

A

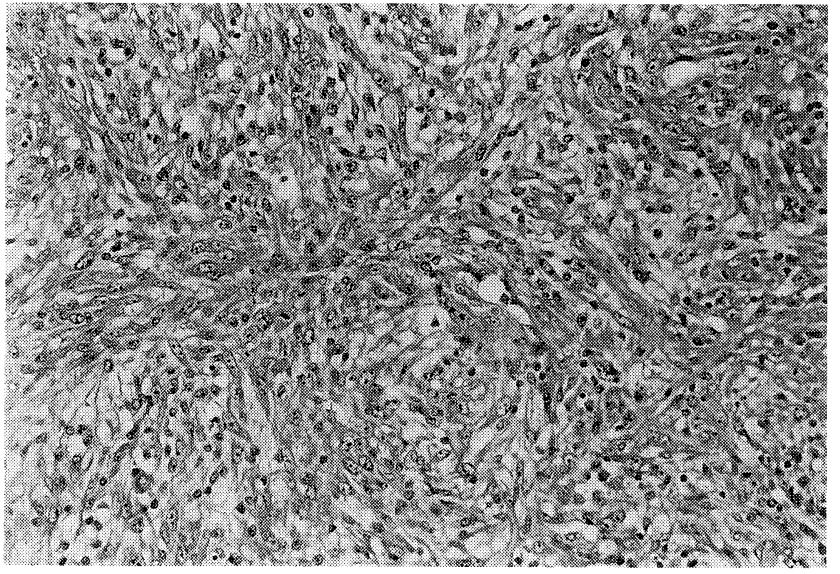

$\mathrm{B}$

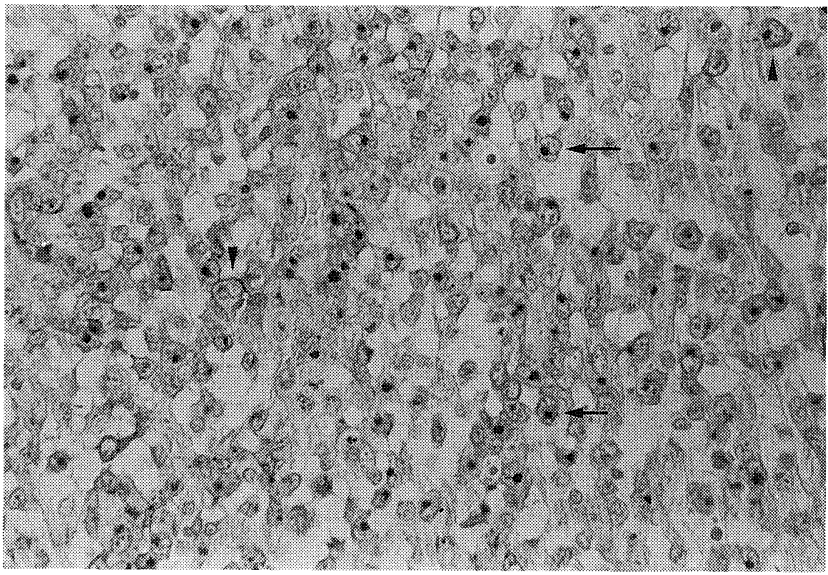

$\mathrm{C}$

Figure 2. Pathological finding of the inguinal mass shows a proliferation of large oval pleomorphic tumor cells with abundant cytoplasm, and the presence of an abundant extracellular myxoid matrix (A: HE stain, $\times$ 400). Some neoplastic cells were spindleshaped with a storiform growth pattern $(B$ : HE stain, $\times 200)$. The stain of $\mathrm{Ki}-1 / \mathrm{Ber}-\mathrm{H} 2$ (CD30) was localized to the cell membrane (arrowhead), Golgi zone (arrow), or both in the tumor cells (C, $\times \mathbf{4 0 0})$. 


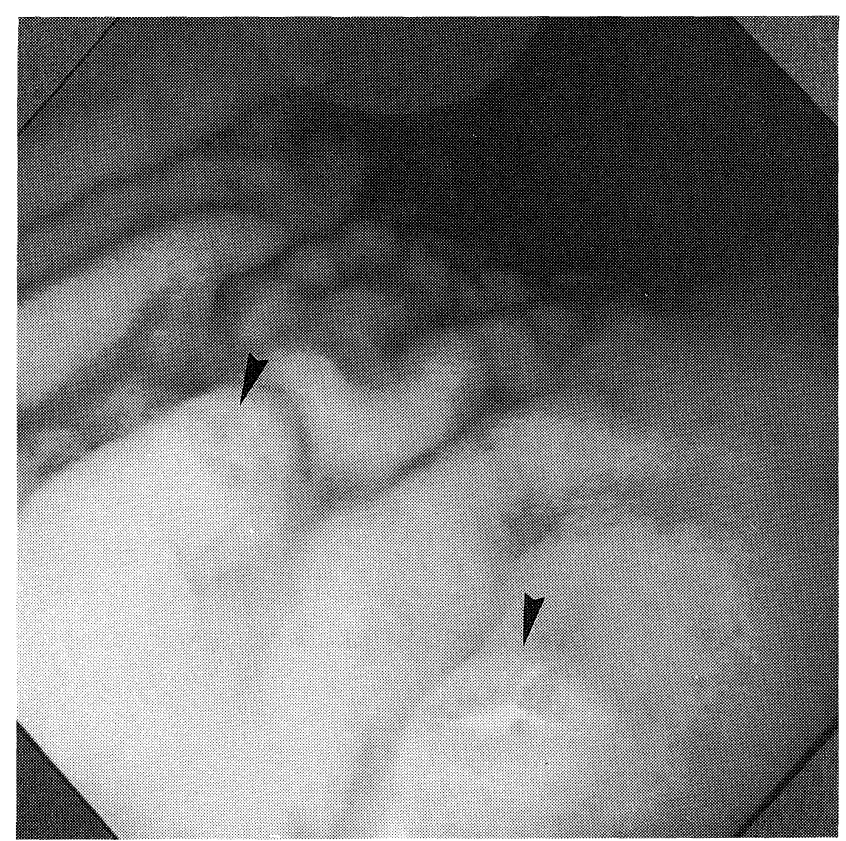

A

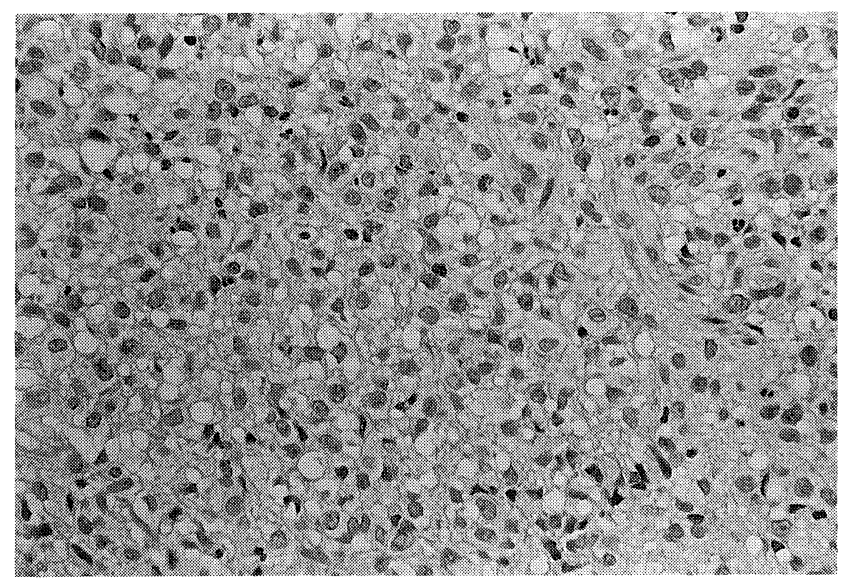

B

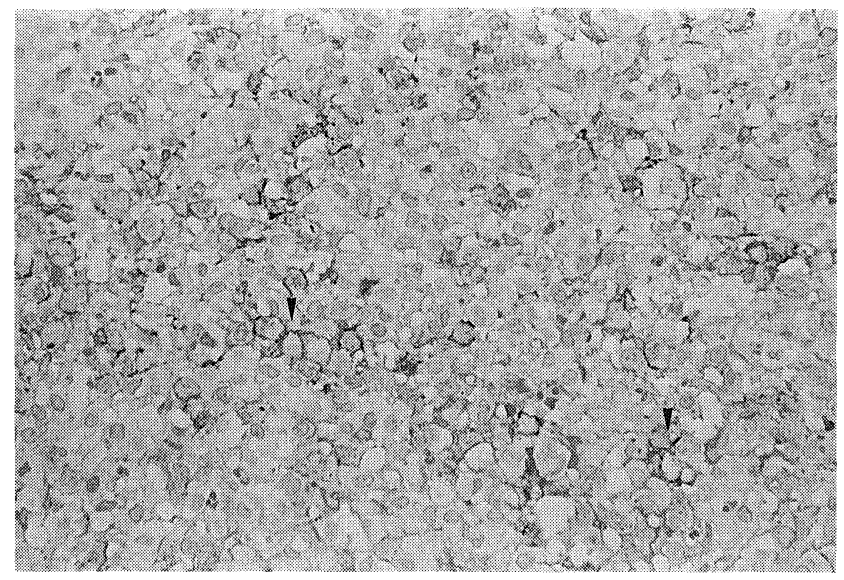

C were not done. The features and immunohistochemical studies were considered to be the Ki-1 (CD30)-positive ALCL of the Tcell type, a sarcomatoid variant.

Transbronchial lung biopsy failed to obtain diagnostic material. However gastric endoscopy showed multiple elevated tumors with dimples (Fig. 3A). Pathological findings of the specimens obtained from the gastric tumors revealed the same results as those of the inguinal mass (Fig. 3B). Immunohistochemical studies showed the staining by $\mathrm{Ki}-1 / \mathrm{Ber}-\mathrm{H} 2$ was localized to only the cell membrane of tumor cells (Fig. 3C).

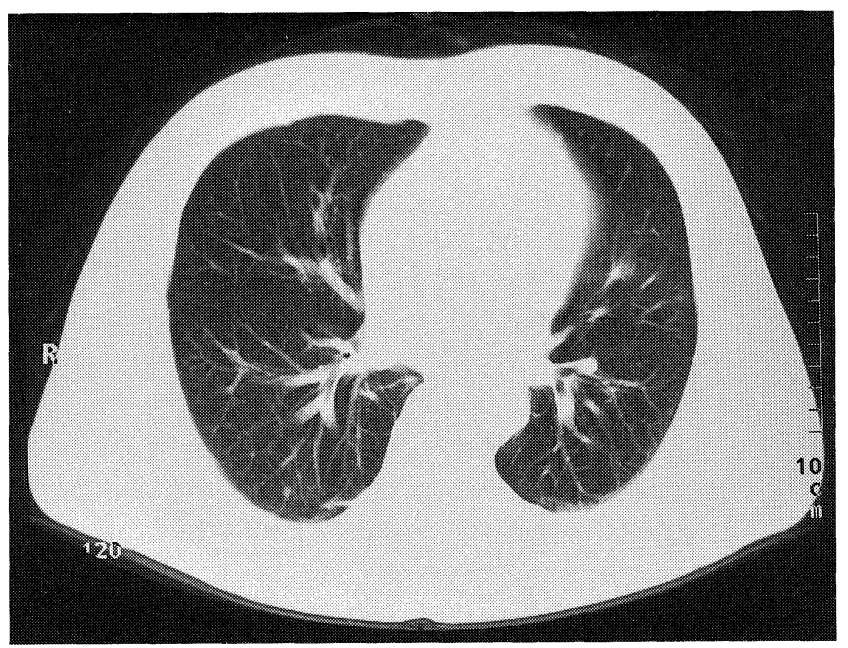

A

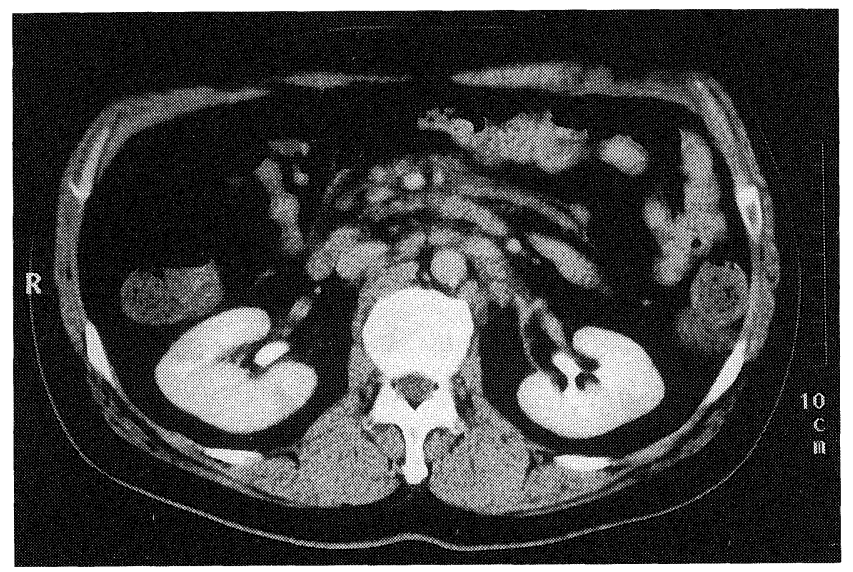

B

Figure 4. Chest (A) and abdominal CT (B) after one month shows that the multiple small nodules of the lung have decreased and the lymphadenopathy has improved.

Figure 3. Gastric endoscopy shows multiple elevated tumors (arrowheads) with dimples (A). Pathological finding of the gastric tumors revealed the same result as in the inguinal mass (B: $\mathrm{HE}$ stain, $\times 400)$. Immunohistochemical studies showed the staining by $\mathrm{Ki}-1 / \mathrm{Ber}-\mathrm{H} 2$ was localized to only the cell membrane (arrowheads) of the tumor cells $(\mathrm{C}, \times \mathbf{4 0 0})$. 
Low-grade fever persisted for about two weeks. Subsequently the fever declined and the inguinal and supraclavicular lymphadenopathy improved gradually during a period of one month without any treatment. At the same time, chest and abdominal CT showed that the lymphadenopathy and multiple small nodules in the lung also decreased in size (Fig. 4A, B). The soluble IL-2R level was restored to the normal range. The patient was discharged with no enlargement of the lymph nodes and no recurrence of the multiple small nodules of the lung. However, he was readmitted two months after his discharge because of recurrence of ALCL including the lymphadenopathy and has been treated with combination chemotherapy (CHOP regimen).

\section{Discussion}

The Ki-1 (CD30) antigen is expressed by not only activated cells in non-neoplastic lymphoid tissues but tumor cells of Hodgkin's disease and various non-Hodgkin's lymphoma (7). ALCL is characterized by an increased number of the Ki-1 antigen positive cells and was incorporated into the updated Kiel classification (8). In ALCL, it has been reported that staining of $\mathrm{Ki}-1 / \mathrm{Ber}-\mathrm{H} 2$, which are also antibodies to designate CD30, is localized to the cell membrane, Golgi zone, or both (9). ALCL histopathologically is characterized by preferential paracortical involvement of lymph nodes, foci of necrosis and intrasinusoidal dissemination of large atypical cells with pleomorphic nuclei, abundant cytoplasm and one or more nucleoli (10). In addition, the sarcomatoid variant (11) has been reported as one of the several morphologic variants of ALCL and corresponds to the histopathological findings of the present case. Chan et al (11) reported that the presence of a storiform or myxoid pattern does not disqualify the diagnosis of malignant lymphoma. With regard to immunophenotypic studies, the tumor cells express EMA, CD25, HLA-DR and CD45 $(2,11)$. It has been reported that expression of EMA and CD45, and the lack of CD15 facilitate the distinction of ALCL from Hodgkin's disease and that the expression of CD30 and a lack of cytokeratin could distinguish ALCL from most carcinomas $(10,12)$.

ALCL has been reported to show frequent extramedullary involvement of the skin, liver, stomach, kidney, bone and lung (13-15). Although ALCL generally has an aggressive clinical behavior, as in the case of other high-grade malignant lymphomas, this disease limited to the skin (primary cutaneous ALCL) tends to have a slower and protracted course (14). The present case had lymphadenopathy, gastric involvement and multiple small nodular shadows in both lung fields. Although transbronchial lung biopsy was of no diagnostic value due to the smallness of the lung nodules, we suspected that the pulmonary lesions indicated involvement of ALCL because they also improved with spontaneous improvement of the lymphadenopathy. It has been reported that primary ALCL of the skin may undergo spontaneous regression (3-5), however there has only been one report (6) in which ALCL without cutaneous involvement showed spontaneous regression of lymphadenopathy. The patient was a 71-year-old female who presented with recurring cervical lymphadenopathy over a 5-year period. Dermierre et al (16) reported that the spontaneous regression occurred in lymphomas, including cutaneous ALCL, especially in cases which were borderline between benign and malignant disease. In addition, Motley et al (17) suggested that regressing atypical histiocytosis is the regressing phase of ALCL of the skin. However, it is unknown why ALCL patients without cutaneous involvement, which is classified as high-grade malignant lymphoma, have spontaneously regressing lymphadenopathy.

An optimal strategy for the treatment of ALCL has not yet been established. The responses to treatment of ALCL limited to the skin are different from those of patients with nodal or other extranodal involvements $(5,14)$. Primary ALCL of the skin can often be controlled by local measures, and intensive treatment does not seem justified $(14,18)$. On the other hand, aggressive chemotherapy should be selected for ALCL with nodal or extranodal involvement, even if the patient has spontaneously regressing lymphadenopathy.

Acknowledgements: The authors are grateful to Prof. J. Patrick Barron of the International Medical Communications Center of Tokyo Medical College for his review of this manuscript.

\section{References}

1) Stein H, Mason DY, Gerdes J, et al. The expression of the Hodgkin's disease associated antigen $\mathrm{Ki}-1$ in reactive and neoplastic lymphoid tissue: evidence that Reed-Sternberg cells and histiocytic malignancies are derived from activated lymphoid cells. Blood 66: 848-858, 1985.

2) Delsol G, Al Saati T, Gatter KC, et al. Coexpression of epithelial membrane antigen (EMA), Ki-1, and interleukin-2 receptor by anaplastic large cell lymphomas. Diagnostic value in so-called malignant histiocytosis. Am J Pathol 130: 59-70, 1988.

3) Chan JK, Ng CS, Hui PK, et al. Anaplastic large cell Ki-1 lymphoma. Delineation of two morphological types. Histopathology 15: 11-34, 1989 (see comments).

4) Bittencourt AL, Mendonca N, de Freitas LA, Schnitzer B. Ki-1 large cell lymphoma with regressing lesions in a child. Pediatr Dermatol 9: 117122,1992

5) Yashiro N, Kitajima J, Kobayashi H, et al. Primary anaplastic large cell lymphoma of the skin. A case report suggesting that regressing atypical histiocytosis and lymphomatoid papulosis are subsets. J Am Acad Dermatol 30: 358-363, 1994.

6) Ragbeer M. Recurrent cervical lymphadenopathy due to Ki-1 lymphoma in an elderly female. Ultrastruct Pathol 19: 383-387, 1995.

7) Miyake K, Yoshino T, Sarker AB, Teramoto N, Akagi T. CD30 antigen in non-Hodgkin's lymphoma. Pathol Int 44: 428-434, 1994.

8) Stansfeld AG, Diebold J, Noel H, et al. Updated Kiel classification for lymphomas. Lancet 1: 292-293, 1988 (letter) (published erratum appears in Lancet 1: 372, 1988).

9) Schwarting R, Gerdes J, Dürkop H, Falini B, Pileri S, Stein H. BER-H2: A new anti-Ki-1 (CD30) monoclonal antibody directed at a formalresistant epitope. Blood 74: 1678-1689, 1989.

10) Kadin ME. Primary Ki-1-positive anaplastic large-cell lymphoma: A distinct clinicopathological entity. Ann Oncol 5 Suppl 1: 25-30, 1994.

11) Chan JK, Buchanan R, Fletcher CD. Sarcomatoid variant of anaplastic large-cell Ki-1 lymphoma. Am J Surg Pathol 14: 983-988, 1990 (see comments).

12) Agnarsson BA, Kadin ME. Ki-1 positive large cell lymphoma. A morphologic and immunologic study of 19 cases. Am J Surg Pathol 12: 264-274, 1988.

13) Penny RJ, Blaustein JC, Longtine JA, Pinkus GS. Ki-1-positive large cell 


\section{ALCL with Regressing Lymphadenopathy}

lymphomas, a heterogenous group of neoplasms. Morphologic, immunophenotype, genotypic, and clinical features of 24 cases. Cancer 68: 362-373, 1991.

14) de Kan R, van't Veer MB. Clinical features of CD30 (Ki-1) positive anaplastic large-cell lymphoma (ALCL). Review of the literature. Neth J Med 43: 277-284, 1993.

15) Kobayashi $T$, Nagasawa $T$, Mitsuhashi $S$, et al. Clinicopathological characteristics of $\mathrm{Ki}-1$ anaplastic large cell lymphoma. Int J Hematol 63: 41-50, 1996.

16) Demierre MF, Goldberg LJ, Kadin ME, Koh HK. Is it lymphoma or lymphomatoid papulosis? J Am Acad Dermatol 36: 765-772, 1997.

17) Motley RJ, Jasani B, Ford AM, Poynton CH, Calonje-Daly JE, Holt PJ. Regressing atypical histiocytosis, a regressing cutaneous phase of $\mathrm{Ki}-1$ positive anaplastic large cell lymphoma. Immunocytochemical, nucleic acid, and cytogenetic studies of a new case in view of current opinion. Cancer 70: 476-483, 1992.

18) Kaudewitz P, Stein H, Dallenbach F, et al. Primary and secondary cutaneous $\mathrm{Ki}-1+(\mathrm{CD} 30+)$ anaplastic large cell lymphomas. Morphologic, immunohistologic, and clinical-characteristics. Am J Pathol 135: 359-367, 1989. 